\title{
ROLE OF IMMUNOTHERAPY IN THE TREATMENT OF TUBERCULOSIS
}

\author{
MURTHY, K.J.R. VIJAYA LAKSHMI, V. and Singh, S. \\ Bhagwan Mahavir Medical Research Centre, 10-1-1, Mahavir Marg, Hyderabad - 500029 . India.
}

\begin{abstract}
Tuberculosis is caused by Mycobacterium tuberculosis, an intracellular pathogen residing in macrophages. Cell mediated immune (CMI) and delayed type of hypersensitive (DTH) responses play a pivotal role in providing protection to the host. The most important cell is the CD4 T lymphocyte, which is divided into TH1 and TH2 subsets depending on the type of cytokines produced. TH1 cells produce the cytokines interferon-gamma and interleukin-2, which are important for activation of antimycobacterial activities and essential for the DTH response. Grange opines that the immune response in an individual with tuberculous infection gets locked in' to one or other pattern of response viz. TH1 or TH2 response, the latter response leading to tissue damage and progression of disease.

Stanford and co-workers conducted several studies on the effectiveness of Mycobacterium vaccae, as an immunotherapeutic agent for tuberculosis. It is non-pathogenic in humans and is thought to be a powerful TH1 adjuvant.

A series of small studies pointed that $M$. vaccae has a beneficial effect and there is enough evidence now to show that its use as an immunotherapeutic agent, as an adjunct to chemotherapy in the treatment of tuberculosis especially at a time when drug resistance is rampant, appears promising.
\end{abstract}

KEY WORDS : Tuberculosis, Drug-Resistance, Immunotherapy, T Cell Responses.

\section{ROLE OF IMMUNOTHERAPY IN THE TREATMENT OF TUBERCULOSIS}

M.tuberculosis is an intracellular pathogen, residing in macrophages, which when activated display microbicidal activity and kill the bacilli. Macrophages are also stimulated by $T$ lymphocytes, which secrete cytokines. T lymphocytes, specific for M.tuberculosis are of paramount importance in both cell mediated immune (CMl) and delayed type of hypersensitive (DTH) responses. DTH response provides some protection, although it results in localised inflammatory responses causing tissue damage.

$T$ cells are important since in addition to macrophages, they also recruit and activate other non-specific inflammatory cells, by the secretion of cytokines. Generally CD4+ T cells are involved, while few CD8 cells participate in the response. TH1 and TH2 are subsets of CD4 lymphocytes, the former playing a pivotal role in providing protection against the infection (1). The important

Address for correspondence :

Dr. K.J.R. Murthy at above address cytokines secreted by the $\mathrm{TH} 1$ cell are interferongamma (IFN- $\gamma$ ) and interleukin-2 (IL-2).

The protection provided by CD4+ cells is well appreciated in the individuals affected with AIDS, since depletion of this cell results in the loss of DTH and low levels of IL-2 and IFN- $\%$. M.tuberculosis is one of the most common oppurtunistic pathogens infecting patients with AIDS.

Defective cell mediated immunity in tuberculosis could be attributed to multiple defects in macrophages or monocytes in patients, such as low phagocytosis or low bacteriocidal properties; defective chemotaxis (attraction of cells to the site of action); presence of suppressor mononuclear cells and/or depletion of CD4+ $T$ iymphocytes resulting in decreased secretion of IFN- $\gamma$ and IL-2.

In a small study conducted at our centre on the specific cell mediated immune response in children, the results suggested that those with tuberculosis (meningitis, lymphadenitis and miliary forms) had significantly low CD4 to CD8 cell ratio, low levels of IFN- $\gamma$ and IL-2, when compared to normal, age and sex-matched group of children (unpublished data). 
Augmentation of depressed cell mediated immune response particularly of the beneficial type (such as $\mathrm{TH} 1$ cell) in immunocompromised individuals like a tuberculosis patient, could go a long way in the treatment of tuberculosis, thereby reducing spread of the disease. Appropriate cellular immune response could perhaps be modulated or stimulated by the use of immunotherapeutic agents.

The concept of immunotherapy for tuberculosis was first exploited by Robert Koch about a 100 years ago (1). He administered repeated injections of old tuberculin. In the 1930's, Spahlinger attempted the use of a slightly modified Koch's therapy. Later, M.chelonae was used as a therapeutic agent but was found to be beneficial only in some cases, particularly in nonpulmonary forms where the bacillary load was small.

In the recent years, Stanford and co-workers conducted several studies on the usefulness of Mycobacterium vaccae as an immunotherapeutic agent for tuberculosis. The main reason bekind the choice of this species, was that it is nonpathogenic to humans. The other reasons include its association with protection because of the presence of group i common mycobacterial antigens, lack of pathogenic significance due to the presence of species specific group iv antigens and its antagonistic features to tissue necrotic elements of Koch phenomenon. M.vaccae is a scotochromogen and a rapidly growing species.

Studies with $M$. vaccae as an immunotherapeutic agent in the treatment of tuberculosis by Stanford and co-workers suggested that immune recognition of common mycobacterial antigens was restored in patients who were previously anergic to the same in skin test. M.vaccae also regulated tissue necrotising immune reactions. In general, the cure rate in newly diagnosed patients was improved.

A higher number i.e. $86 \%$ of patients in the immunotherapy group, compared to $58 \%$ of the placebo group, showed progressive resolution of cavities. This improved radiological appearance associated with immunotherapy was noted in an early study of M. vaccae in Kuwait (1). Similar results were observed in pilot studies in Argentina, Romania and Vietnam (1). In Nigeria, in patients with HIV related tuberculosis, increased survival and sputum clearance was attributed to immunotherapy with M.vaccae. Also there was resolution of generalised lymphadenopathy associated with HIV infection Two patients who received a placebo were still positive for HIV antibody, one year later, whereas, two others who received immunotherapy with $M$. vaccae, tested negative, after one year. The results suggested that $M$. vaccae evokes the beneficial type of response.

The mode of action of M.vaccae, according to Stanford and group is on the pathway of $T$ helper cell maturation, production of type 1 cytokines and regulation of immunopathology (2). Grange proposed the theory that the immune response in an individual with tuberculous infection gets locked in' to one or other pattern of response, viz. TH1 or TH2 response (3). The latter is an inappropriate response leading to tissue damage and progression of disease. M. vaccae is a powerful $\mathrm{TH} 1$ adjuvant, perhaps supressing TH2 mediated responses (1).

Improvement of immunity and stimulation of the relevant responses in patients with tuberculosis by immunotherapy, is not only encouraging, but raises hopes in the treatment of drug-resistant tuberculosis, where chemotherapy alone is not effective in the elimination of bacteria.

Drug-resistant tuberculosis is a cause for concern as its incidence is now on the rise. Resistance to one, two or more drugs is becoming more common. The problems, present in the preantibiotic era of tuberculosis, have surfaced once again.

In India, an increase in acquired rifampicinresistance was reported by ICMR in 1968 after a surveillance. In Gujarat, a rise from $2.8 \%$ in 1980 to $37.3 \%$ in 1986 was observed (4). Local statistics reveal that $16.1 \%$ of patients with tuberculosis are resistant to more than two drugs (Goud \& Chandrasekhar, 1997, personal communication). Infection with MDR-strains and outbreak of such bacilli, may become a common phenomenon in patients with AIDS. Some strains of tubercle bacilli are resistant to seven or more antibiotics.

In a small study conducted in Iran (5), clinical cure and bacteriological resolution was observed in $27 \%$ of multi-drug resistant tuberculous patients, whilst only $1 \%$ were cured with chemotherapy alone (5). In a recent report, 13 out of 14 patients, seven with MDR-TB, were cured. A preliminary study conducted at our centre, on the use of $M$. vaccae as an adjunct to chemotherapy, in 
patients with MDR-TB, showed clearance of bacilli, increase in body weight and $\mathrm{CMI}$ response in almost all the patients.

Observations from several studies indicated that M.vaccae had beneficial effects when used as an adjunct to chemotherapy. However, the ideal time and number of times it has to be administered for the patients to obtain optimum benefit, is yet to be established. While a single injection was sufficient in patients with a short history of less than two years of chemotherapy, repeated injections were required for chronic patients with longer treatment history. Thus $86 \%$ patients with primary resistance were cured after a single injection, while $22 \%$ with chronic disease and acquired resistance were cured only after multiple (2-4) injections of M.vaccae (6). While, in a study in Romania, in patients who had histories of disease longer than two years, there was a significantly improved cure rate by a single injection. At present, in the phase III trial being conducted at Durban, M.vaccae is injected on the day 8 of treatment and in a study in London it is being injected during the first three days of treatment. The authors feel that further benefit from immunotherapy could be obtained by even adminisiering it on the first day of chemotherapy (6).

Thus, in tuberculous infection, elimination of bacilli, eventually leading to cure depends largely on the immune response of the individual, particularly the cell mediated. The disease manifests in a host who is immunocompromised. Intervention by chemotherapy further assists in the elimination of the pathogen, especially the persistors. The idea of enhancing the relevant immune responses by immunotherapy is, appealing. There is enough evidence now to show that immunotherapy with Mycobacterium vaccae, as an adjunct to chemotherapy, is effective. Therefore, eradication of tuberculosis which seems like an uphill task now, may not be a far-fetched idea after all.

Table 1 : Changes in \% LMI (Leucocyte Migration Inhibition) with PPD, weight, sputum conversion in MDR-TB-patients, 3-6 months after receiving immunotherapy with $M$. vaccae

(all the patients, barring one, had positive \% LMI values to PHA-a mitogen) : $(B=$ Before immunotherapy; $A=$ After immunotherapy; $N C=$ No Change)

\begin{tabular}{|c|c|c|c|c|c|c|}
\hline \multirow[t]{2}{*}{ sl..No. } & \multicolumn{2}{|c|}{$\%$ LMI (PPD) } & \multicolumn{2}{|c|}{ Weight } & \multicolumn{2}{|c|}{ Sputum conversion } \\
\hline & $\overline{\mathrm{B}}$ & $\bar{A}$ & $\overline{\mathrm{B}}$ & $\bar{A}$ & $B$ & $\bar{A}$ \\
\hline 1. & 30 & +10 & 34 & NC & $(+)$ & $(-)$ \\
\hline 2. & 18 & +2 & 60 & +2 & $(+)$ & $(-)$ \\
\hline 3. & 16 & +10 & 49 & +3 & $(+)$ & $(-)$ \\
\hline 4. & 13 & +17 & 40 & NC & $(++)$ & $(-)$ \\
\hline 5. & 10 & +30 & 54 & -5 & $(++)$ & $(+)$ \\
\hline 6. & 13 & +10 & 39 & NC & $(+)$ & $(-)$ \\
\hline 7. & 6 & +14 & 60 & +5 & $(++t)$ & $(-)$ \\
\hline 8. & 28 & -1 & 51 & +2 & $(+)$ & $(+)$ \\
\hline 9. & 35 & -10 & 53 & -9 & $(+)$ & $(-)$ \\
\hline 10. & 16 & +9 & 36 & +4 & $(+)$ & $(-)$ \\
\hline 11. & 21 & +3 & -- & -- & $(+)$ & $(+)$ \\
\hline 12. & 28 & +12 & 78 & +2 & $(+)$ & $(-)$ \\
\hline
\end{tabular}

\section{REFERENCES}

1. Stanford J.L. and Grange J.M. (1994) The promise of immunotherapy for tuberculosis. Respir. Med. 88, 3-7. 
2. Corlan E., Marica, C., Macavei, C., Stanford, J.L. and Stanford, C.A. (1997) Immunotherapy with Mycobacterium vaccae in the treatment of tuberculosis in Romania. 1. Newly-diagnosed pulmonary disease. Respir. Med. 91, 13-19.

3. Grange, J.M. (1997) Immunotherapy : A new strategy for tuberculosis control Respir. Med. 91, $1-4$.

4. Jain N.K. (1992) Drug resistance in India : A tragedy in the making. Ind. J.Tub. 39, 145-148.

5. Farid, R., Etemadi, A., Mehvar, M., Stanford, J.L., Dowlati, Y. and Velayati, A.A. (1994) Mycobacterium vaccae immunotherapy in the treatment of multi-drug-resistant tuberculosis : A preliminary Report. Irn. J. Med. Sci. 19 (182), 37-39.

6. Stanford, J.L. and Stanford, C.A. (1996) Immunotherapy with Mycobacterium vaccae and the treatment of tuberculosis. J. Appl. Bacteriol $81,81 \mathrm{~S}-86 \mathrm{~S}$. 